\title{
Toy model of boundary states with spurious topological entanglement entropy
}

\author{
Kohtaro Kato ${ }^{1}{ }^{1}$ and Fernando G. S. L. Brandão $0^{1,2}$ \\ ${ }^{1}$ Institute for Quantum Information and Matter, California Institute of Technology, Pasadena, California 91125, USA \\ ${ }^{2}$ Amazon Web Services, AWS Center for Quantum Computing, Pasadena, California 91125, USA
}

(Received 21 February 2020; revised 27 April 2020; accepted 29 April 2020; published 7 July 2020)

\begin{abstract}
Topological entanglement entropy has been extensively used as an indicator of topologically ordered phases. We study the conditions needed for two-dimensional topologically trivial states to exhibit spurious contributions that contaminate topological entanglement entropy. We show that, if the state at the boundary of a subregion is a stabilizer state, then it has a nonzero spurious contribution to the region if and only if the state is in a nontrivial one-dimensional $G_{1} \times G_{2}$ symmetry-protected-topological (SPT) phase under an on-site symmetry. However, we provide a candidate of a boundary state that has a nonzero spurious contribution but does not belong to any such SPT phase.
\end{abstract}

DOI: 10.1103/PhysRevResearch.2.032005

\section{INTRODUCTION}

Topologically ordered phases are gapped quantum phases that cannot be detected by conventional local order parameters. Topological entanglement entropy (TEE) $[1,2]$ has been widely used as an indicator of such phases. For ground states in gapped two-dimensional (2D) models, the entanglement entropy $S(A):=-\operatorname{Tr} \rho_{A} \log _{2} \rho_{A}$ of a region $A$ is expected to behave as

$$
S(A)=\alpha|\partial A|-\gamma+o(1)
$$

where $\alpha$ is a constant, $\partial A$ is the boundary length, and $o(1)$ comprises terms vanishing in the limit of $|\partial A| \rightarrow \infty$. TEE is defined as the universal constant term $\gamma$ [1]. The term $\gamma$ is shown to be the logarithm of the total quantum dimension of the abstract anyon model under various conditions [1,3-5].

To extract the TEE from a ground state, one can calculate suitable linear combinations of entropies for certain subsystems [see, e.g., Fig. 1(a)], known as conditional mutual information (CMI) in quantum information theory, such that the first leading terms cancel out [1,2].

However, in general, Eq. (1) could contain an additional term, and thus the above argument does not always work. This additional contribution, called spurious TEE [6,7], results in positive CMI for states in the trivial phase.

Thus far, the spurious TEE seems to be connected to the existence of a one-dimensional (1D) symmetry-protectedtopological (SPT) phase at the boundary of a certain region [6-10]. Spurious TEE appears fragile against general local perturbations or small deformation of the regions, but the

Published by the American Physical Society under the terms of the Creative Commons Attribution 4.0 International license. Further distribution of this work must maintain attribution to the author(s) and the published article's title, journal citation, and DOI. conditions under which spurious TEE appears are yet to be fully understood.

A natural question is whether a SPT phase at the boundary is also a necessary condition for spurious TEE. In this paper, we study the underlying mechanism behind spurious TEE in the trivial phase. We model the degrees of freedom at the boundaries of regions [Fig. 1(b)] by using matrixproduct states (MPSs) [11]. Here, we focus in particular on a renormalization fixed point of the MPS in which the CMI is constant for all length scales. We then characterize the fixed points in terms of the operator-algebra quantum-error correction (OAQEC) [12-14] and derive a formula to calculate the value of the spurious TEE from algebras associated with the single tensor.

Using our characterization, we show that, if the boundary MPS is a stabilizer state [15], a nonzero spurious TEE implies that the MPS is in a nontrivial $G_{1} \times G_{2}$ SPT phase under onsite symmetry actions. By contrast, we also provide numerical evidence that, in general, there exist boundary states that have nonzero spurious TEE but do not belong to any such SPT phase. To the best of our knowledge, this is the first example of the mechanism of spurious TEE beyond the on-site $G_{1} \times G_{2}$ SPT phase at the boundary.

\section{MATRIX-PRODUCT-STATE MODEL OF BOUNDARY STATES}

We consider a translation-invariant ground state $|\psi\rangle$ defined on a 2D spin lattice of size $N$. When a ground state is in the trivial phase, it can be (approximately) constructed from a product state only by a constant-depth local unitary circuit $[16,17]$. More precisely, there exists a set of unitaries $\left\{V_{i}\right\}$ such that

$$
|\psi\rangle=V_{w} V_{w-1} \cdots V_{1}|0\rangle^{\otimes N},
$$

where the depth $w=\mathcal{O}(1)$ is a constant of $N$ and each $V_{i}$ is a product of local unitaries acting on disjoint sets of neighboring spins within radius $r=\mathcal{O}(1)$. 
(a)

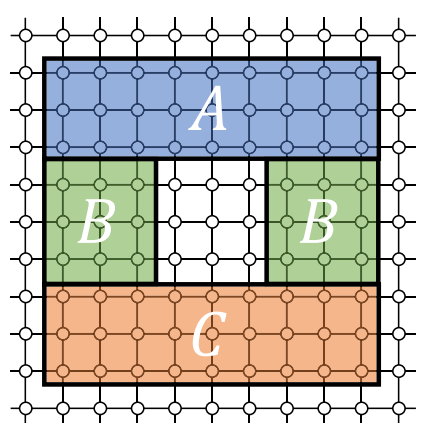

(b)

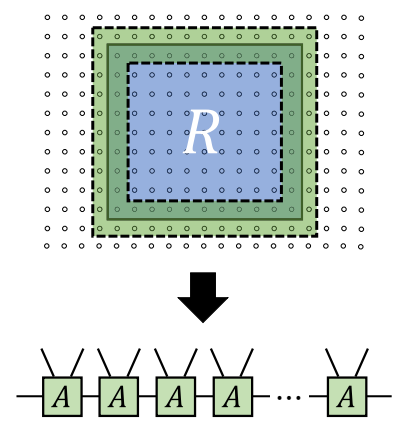

FIG. 1. (a) A tripartition of a subsystem in a 2D spin lattice to calculate the TEE. (b) For ground states in the trivial phase, the entanglement entropy of region $R$ is determined by a MPS located at the boundary of $R$ (green region). Each tensor $A$ has two physical legs associated with $R$ and its complement, respectively.

Let us divide the lattice into a connected region $R$ and its complement $R^{c}$. Entanglement between $R$ and $R^{c}$ is invariant under local unitaries $U_{R} U_{R^{c}}$; therefore, we can undo some parts of the circuit. Hence, $S(R)_{\rho}$ is equivalent to that of a tensor product of an entangled state $|\phi\rangle_{R R^{c}}$ around the boundary $\partial R$ and $|0\rangle$ s elsewhere. We call $|\phi\rangle_{R R^{c}}$ the boundary state of $R$ [Fig. 1(b)].

A constant-depth circuit can increase the Schmidt-rank by at most a constant. Therefore $|\phi\rangle_{R R^{c}}$ is written as a MPS [Fig. 1(b)]:

$$
|\phi\rangle_{R R^{c}}=\sum \operatorname{Tr}\left(A^{i_{1} j_{1}} \ldots A^{i_{l} j_{l}}\right)\left|i_{1}, \ldots, i_{l}\right\rangle_{R}\left|j_{1}, \ldots, j_{l}\right\rangle_{R^{c}},
$$

where $A^{i_{k} j_{k}}$ is a $D \times D$ matrix with a constant bond dimension $D=\mathcal{O}(1)$. Here, we assume that all the tensors are the same due to the translation-invariance. ${ }^{1}$ Each local basis $\left\{\left|i_{k}\right\rangle\right\}$ corresponds to a coarse-grained site consisting of several neighboring spins so that the correlation length of the MPS is exactly zero. We use $\mathcal{H}$ and $\mathcal{K}$ to denote the Hilbert spaces associated with $|i\rangle_{R}$ and $|j\rangle_{R^{c}}$, respectively. In this notation, there is an isometry $V: \mathbb{C}^{D} \otimes \mathbb{C}^{D} \rightarrow \mathcal{H} \otimes \mathcal{K}, V^{\dagger} V=I$, such that the MPS has the form $[11,18]$

$$
|\phi\rangle_{R R^{c}}=V^{\otimes l}\left|\lambda_{D}\right\rangle^{\otimes l},
$$

where $\left|\lambda_{D}\right\rangle=\sum_{k=1}^{D} \sqrt{\lambda_{k}}|k k\rangle$ is an entangled state with the Schmidt rank $D . V$ acts on two separated sites of neighboring $|\lambda\rangle$ s. In the following, we especially consider the case in which $\left|\lambda_{D}\right\rangle$ is the maximally entangled state $\left|\omega_{D}\right\rangle:=$ $\sum_{i=1}^{D} \frac{1}{\sqrt{D}}|i i\rangle$ for simplicity. We do not expect to lose much generality by this reduction, although we leave extensions for future work.

When $R$ is an annulus like $A B C$ in Fig. 1(a), we obtain two boundary states at the inner and outer boundaries. The ground

\footnotetext{
${ }^{1}$ This assumption is slightly stronger than translation invariance, since generally tensors can depend upon the direction of the edge and can even contain some "corner" tensors. However, such corner contributions cancel out in the calculation of CMI.
}

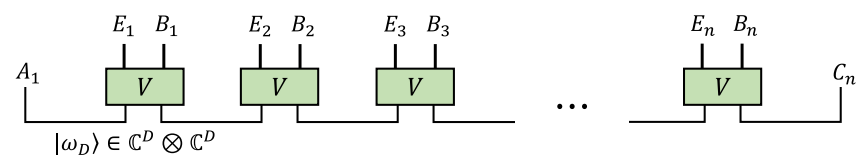

FIG. 2. A schematic picture of the family of states $\phi^{(n)} .\left|\omega_{D}\right\rangle=$ $\sum_{i} \frac{1}{\sqrt{D}}|i i\rangle$ is the $D$-dimensional maximally entangled state and $V$ is an isometry from $\mathbb{C}^{D} \otimes \mathbb{C}^{D}$ to $\mathcal{H} \otimes \mathcal{K}$.

state has a spurious TEE for $R$ if one of these boundary states has a nontrivial CMI

$$
I(A: C \mid B)_{\rho}:=S(A B)+S(B C)-S(B)-S(A B C)>0
$$

for a tripartition $R=A B C$ such that $B$ separates $A$ from $C$. Importantly, the value of CMI matches that of the tri-information [19], which is also used to extract TEE [1] for the class of states that we are considering.

Due to the monotonicity of CMI, a nonzero value of the spurious TEE implies that the CMI of an open-boundary MPS must be positive as well. We formalize a family of such openboundary MPS $\left\{\phi^{(n)}\right\}_{n \geqslant 0}$ with different lengths $n$ defined as

$$
\begin{aligned}
\phi^{(0)} & :=\left|\omega_{D}\right\rangle\left\langle\left.\omega_{D}\right|_{A_{1} A_{2}},\right. \\
\phi^{(n)} & :=\mathcal{V}_{A_{2 n} A_{2 n+1} \rightarrow B_{n} E_{n}}\left(\phi^{(n-1)} \otimes\left|\omega_{D}\right\rangle\left\langle\left.\omega_{D}\right|_{A_{2 n+1} A_{2 n+2}}\right),\right.
\end{aligned}
$$

where $\mathcal{V}_{A_{2 n} A_{2 n+1}}(X)=V X V^{\dagger}$ is the isometry map (Fig. 2). For convenience, we relabel $A_{2 n+2}$ by $C_{n}$ so that each $\phi^{(n)}$ is a state on $A_{1} \otimes\left(B_{1} \otimes E_{1}\right) \otimes\left(B_{2} \otimes E_{2}\right) \otimes \cdots \otimes\left(B_{n} \otimes E_{n}\right) \otimes C_{n} . A_{1}$ and $C_{n}$ represent the unfixed boundary condition.

After tracing out $R^{c}=E_{1} \cdots E_{n}$, we have a family of mixed states $\left\{\rho^{(n)}\right\}_{n \geqslant 0}$ defined by

$$
\begin{aligned}
& \rho^{(0)}:=\left|\omega_{D}\right\rangle\left\langle\left.\omega_{D}\right|_{A_{1} A_{2}},\right. \\
& \rho^{(n)}:=\mathcal{E}_{A_{2 n} A_{2 n+1} \rightarrow B_{n}}\left(\rho^{(n-1)} \otimes\left|\omega_{D}\right\rangle\left\langle\left.\omega_{D}\right|_{A_{2 n+1} A_{2 n+2}}\right),\right.
\end{aligned}
$$

where $\mathcal{E}=\operatorname{Tr}_{E} \circ \mathcal{V}$ is a completely positive and tracepreserving (CPTP) map. We denote the CPTP map $\mathcal{E}(\cdot \otimes$ $\left.\left|\omega_{D}\right\rangle\left\langle\omega_{D}\right|\right)$ by $\tilde{\mathcal{E}}$. We then have

$$
\rho^{(n+1)}=\tilde{\mathcal{E}}_{C_{n} \rightarrow B_{n+1} C_{n+1}}\left(\rho^{(n)}\right) .
$$

The whole family is obtained by iteratively applying $\tilde{\mathcal{E}}$ :

$$
\rho^{(n)}=\tilde{\mathcal{E}}_{C_{n} \rightarrow B_{n+1} C_{n+1}} \circ \cdots \circ \tilde{\mathcal{E}}_{A_{2} \rightarrow B_{1} C_{1}}\left(\rho^{(0)}\right)
$$

(recalling that $C_{0}=A_{2}$ ). We will simply denote the concatenated map in Eq. (4) by $\tilde{\mathcal{E}}^{(n)}$. When we trace out $R$ instead of $R^{c}$, we obtain the complement chain, which we denote by $\left\{\sigma^{(n)}\right\}$. We also define $\mathcal{F}:=\operatorname{Tr}_{B} \circ \mathcal{V}$ and $\tilde{\mathcal{F}}(\cdot)=\mathcal{E}^{c}(\cdot \otimes$ $\left.\left|\omega_{D}\right\rangle\left\langle\omega_{D}\right|\right)$.

$\left\{\phi^{(n)}\right\}$ has a spurious TEE if $I\left(A_{1}: C_{n} \mid B_{1} \cdots B_{n}\right)_{\rho^{(n)}}$ is bounded from below by a positive constant. Although $\rho^{(n)}$ has zero correlation length, we might still have a nontrivial length scale for the CMI [20]. We further remove this length scale by requiring saturation of the $\mathrm{CMI}$ :

$$
I\left(A_{1}: C_{1} \mid B_{1}\right)_{\rho^{(1)}}=I\left(A_{1}: C_{n} \mid B_{1} \cdots B_{n}\right)_{\rho^{(n)}} \quad \forall n .
$$

Note that the left-hand side (LHS) is always larger than or equal for any CPTP-map $\mathcal{E}$. In the rest of this paper, we simply denote LHS by $I\left(A_{1}: C_{n} \mid B_{1} B_{2} \cdots B_{n}\right)_{(n)}$.

While definition (5) depends on $n$, it is equivalent to two independent conditions independent of $n$. 
Proposition 1. Equation (5) is equivalent to

$$
\begin{gathered}
I\left(A_{1}: B_{1} C_{1}\right)_{(1)}=I\left(A_{1}: B_{1} B_{2} C_{2}\right)_{(2)}, \\
I\left(A_{1}: B_{1}\right)_{(1)}=I\left(A_{1}: B_{1} B_{2}\right)_{(2)} .
\end{gathered}
$$

Moreover, Eq. (7) is equivalent to

$$
I\left(A_{1}: E_{1} C_{1}\right)_{(1)}=I\left(A_{1}: E_{1} E_{2} C_{2}\right)_{(2)} .
$$

Therefore, it is sufficient to consider up to $n=2$. See (Sec. III, [21]) for the proof.

\section{CHARACTERIZATION BY OPERATOR-ALGEBRA QUANTUM-ERROR CORRECTION}

We use the theory of operator-algebra quantum-error correction (OAQEC) [12-14] to characterize $\left\{\phi^{(n)}\right\}$. OAQEC is a general framework for quantum-error correction including standard codes [22] and subsystem codes [23]. It allows us to describe what types of observables are correctable against a given error. For a given CPTP-map $\mathcal{E}: \mathcal{H} \rightarrow \mathcal{K}$ representing a "noise," one can always specify the correctable algebra $\mathcal{A}_{\mathcal{E}} \subset \mathcal{B}(\mathcal{H})$, which is a $C^{*}$ algebra containing all observables whose information is preserved under $\mathcal{E}$ [see Supplemental Material (SM) for more details] [21].

In the following analysis, the correctable algebras of $\tilde{\mathcal{E}}$ and $\tilde{\mathcal{F}}$ play a crucial role. We first show that the saturation of the conditional mutual information (5) implies the saturation of these correctable algebras.

Proposition 2. If Eq. (5) holds for $\mathcal{E}$, then

$$
\begin{aligned}
\mathcal{A}_{\tilde{\mathcal{E}}} & =\mathcal{A}_{\tilde{\mathcal{E}}^{(n)}}, \\
\mathcal{A}_{\tilde{\mathcal{F}}} & =\mathcal{A}_{\tilde{\mathcal{F}}^{(n)}}, \\
\mathcal{A}_{\operatorname{Tr}_{C^{\circ} \tilde{\mathcal{E}}}} & =\mathcal{A}_{\operatorname{Tr}_{C} \circ \tilde{\mathcal{E}}^{(n)}}, \\
\mathcal{A}_{\operatorname{Tr}_{C^{\circ} \circ \tilde{\mathcal{F}}}} & =\mathcal{A}_{\operatorname{Tr}_{C^{\circ} \circ \tilde{\mathcal{F}}}(n)} \forall n .
\end{aligned}
$$

This proposition means that the algebra $\mathcal{A}_{\tilde{\mathcal{E}}}$ represents the information of the input, which is faithfully encoded in the output on $B_{1} \cdots B_{n} C_{n}$ for all $n$. In the same way, $\mathcal{A}_{\operatorname{Tr}_{C} \circ \tilde{\mathcal{E}}}$ represents the perfectly recoverable information encoded on $B_{1} \cdots B_{n}$.

In general, there are operators carrying "unpreserved" information, which are disturbed and cannot be recovered perfectly. Such operators may also contribute to CMI, but may decrease it with respect to $n$. Proposition 2 does not prevent such unpreserved operators and therefore the conditions (9)(12) are insufficient for Eq. (5). In fact, we can always assume these conditions by coarse-graining a finite number of channels. ${ }^{2}$ We utilize the concept of the complementary-recovery property [24] because we intend to neglect this unpreserved information.

\footnotetext{
${ }^{2}$ For any $\mathcal{E}$, we have $\mathcal{B}\left(\mathbb{C}^{D}\right) \supset \mathcal{A}_{\tilde{\mathcal{E}}} \supset \mathcal{A}_{\tilde{\mathcal{E}}^{(2)}} \supset \cdots \supset \mathbb{C} I$, and thus we cannot have an infinitely long sequence of strictly different $C^{*}$ algebras. Therefore, $m \in \mathbb{N}$ exists such that the conditions hold by redefining $\tilde{\mathcal{E}} \equiv \tilde{\mathcal{E}}^{(m)}\left(\mathcal{F} \equiv \tilde{\mathcal{F}}^{(m)}\right)$.
}

Definition 1. We say that a CPTP-map $\mathcal{E}$ satisfies the complementary-recovery property if

$$
\mathcal{A}_{\mathcal{E}^{c}}=\mathcal{A}_{\mathcal{E}}^{\prime}
$$

where $\mathcal{A}_{\mathcal{E}}^{\prime}$ is the commutant of $\mathcal{A}_{\mathcal{E}}$.

Any CPTP map satisfies $\mathcal{A}_{\mathcal{E}^{c}} \subset \mathcal{A}_{\mathcal{E}}^{\prime}$, i.e., any operator recoverable from the output of the complementary channel $\mathcal{E}^{c}$ should commute with the correctable algebra of the original channel $\mathcal{E}$ (see also the SM [21]). As per the complementaryrecovery property, the converse of this statement is also true. This property can be characterized by a projection map onto the correctable algebra.

Proposition 3. $\mathcal{E}$ satisfies the complementary-recovery property if and only if

$$
\begin{gathered}
\mathcal{E}\left(\mathcal{P}_{\mathcal{A}_{\mathcal{E}}}(\rho)\right)=\mathcal{E}(\rho) \forall \rho \\
\text { or } \\
\mathcal{P}_{\mathcal{A}_{\mathcal{E}}}^{\dagger} \circ \mathcal{E}^{\dagger}(O)=\mathcal{E}^{\dagger}(O) \quad \forall O,
\end{gathered}
$$

where $\mathcal{P}_{\mathcal{A}_{\mathcal{E}}}^{\dagger}: \mathcal{B}(\mathcal{H}) \rightarrow \mathcal{A}_{\mathcal{E}}$ is a conditional expectation onto $\mathcal{A}_{\mathcal{E}}$.

Therefore, the complementary-recovery property restricts the input information to the correctable algebra.

Definition 2. We say isometry $V$ or CPTP map $\mathcal{E}$ satisfies dual complementarity if $\tilde{\mathcal{E}}$ and $\tilde{\mathcal{F}}$ both satisfy the complementary-recovery property.

Dual complementarity reduces the four algebras in Proposition 2 to two algebras $\mathcal{A}:=\mathcal{A}_{\tilde{\mathcal{E}}}=\left(\mathcal{A}_{T r_{C} \circ \tilde{\mathcal{F}}}\right)^{\prime}$ and $\mathcal{B}:=$ $\mathcal{A}_{\tilde{\mathcal{F}}}=\left(\mathcal{A}_{\operatorname{Tr}_{C} \circ \tilde{\mathcal{E}}}\right)^{\prime}$. In what follows, we only consider states satisfying this property. We show that dual complementarity implies saturation of the CMI. Furthermore, its value is determined by $\mathcal{A}$ and $\mathcal{B}$.

Theorem 1. If $V$ satisfies dual complementarity, then Eq. (5) holds. Let $\mathcal{A}=\bigoplus_{k} M_{n_{k}}(\mathbb{C}) \otimes I_{n_{k}^{\prime}}$ and $\mathcal{B}=\bigoplus_{l} M_{m_{l}}(\mathbb{C}) \otimes I_{m_{l}^{\prime}}$. Then, the value of the CMI is given by

$$
I\left(A_{1}: C_{1} \mid B_{1}\right)_{(1)}=\sum_{k} p_{k} \log _{2} \frac{n_{k}}{n_{k}^{\prime}}+\sum_{l} q_{l} \log _{2} \frac{m_{l}}{m_{l}^{\prime}},
$$

where $p_{k}=\frac{n_{k} n_{k}^{\prime}}{D}$ and $q_{l}=\frac{m_{l} m_{l}^{\prime}}{D}$. Therefore, $I\left(A_{1}: C_{1} \mid B_{1}\right)_{(1)}>$ 0 if and only if

$$
\mathcal{B}^{\prime} \subsetneq \mathcal{A}
$$

Equation (17) intuitively means an operator exists that is perfectly encoded in $B C$ whose information cannot be read out by just looking $B$. Such nonlocal information causes a spurious contribution to CMI.

Note that dual complementarity is not a necessary condition for Eq. (5). For example, one can consider $\mathcal{E}=\mathcal{E}_{A_{2}}^{\prime} \otimes \mathrm{id}_{A_{3}}$ such that $\mathcal{E}^{\prime}$ is not the completely depolarizing channel, but $\mathcal{A}_{\mathcal{E}^{\prime}}=\mathbb{C} I$. The corresponding family satisfies $I(A: C \mid B)=$ 0 for any length, but the map does not satisfy dual complementarity. This is because all unpreserved information is transferred to $B$ and not $C$, and thus it cancels out in $I(A$ : $C \mid B)=I(A: B C)-I(A: B)$. 


\section{RELATION TO SYMMETRY-PROTECTED- TOPOLOGICAL PHASES}

For any $O \in \mathcal{A}$, we always find a corresponding logical operator $\widetilde{O}_{B C}$ such that

$$
\mathcal{V}_{A_{2} \rightarrow B E C}\left(O_{A_{2}}|\psi\rangle_{A_{2}}\right)=\left(\tilde{O}_{B C} \otimes I_{E}\right) \mathcal{V}_{A_{2} \rightarrow B E C}|\psi\rangle_{A_{2}}
$$

for any $|\psi\rangle \in \mathbb{C}^{D}$, where

$$
\mathcal{V}_{A_{2} \rightarrow B E C}|\psi\rangle_{A_{2}}:=V^{\otimes n}\left(|\psi\rangle_{A_{2}} \otimes \bigotimes_{i}\left|\omega_{D}\right\rangle_{A_{2 i+1} A_{2 i+2}}\right) .
$$

In general, the logical operator is not unique. The set of all logical operators $\mathcal{L}_{\mathcal{A}}$ in $\mathcal{A}$ is given as the pre-image of $\tilde{\mathcal{E}}^{(n) \dagger}$ :

$$
\mathcal{L}_{\mathcal{A}}:=\left\{O_{B C} \mid \tilde{\mathcal{E}}^{(n) \dagger}\left(O_{B C}\right) \in \mathcal{A}\right\} .
$$

$\tilde{\mathcal{E}}^{(n) \dagger}$ is a normal $*$ homomorphism from the pre-image to $\mathcal{A}$ [14]. By the first isomorphism theorem for algebra, the image of the homomorphism is isomorphic to the pre-image up to the kernel:

$$
\mathcal{L}_{\mathcal{A}} / \operatorname{Ker} \tilde{\mathcal{E}}^{(n) \dagger} \cong \mathcal{A} .
$$

We denote the equivalence class of the logical operators of $O \in \mathcal{A}$ by $\mathcal{L}(O)$.

Suppose that the boundary state is in a nontrivial SPT phase under a symmetry of group $G_{1} \times G_{2}$ acting on each tensor as $U\left(g_{1}, g_{2}\right)=U\left(g_{1}\right)_{B} \otimes U^{\prime}\left(g_{2}\right)_{E}$. The action induces a projective representation $V(g) \otimes V(g)^{\dagger}$ on the virtual degrees of freedom [25] (see also the SM [21]). For instance, it holds that

$$
U(g)_{B_{1} E_{1}}\left|\phi^{(1)}\right\rangle_{A_{1} B_{1} E_{1} C_{1}}=V(g)_{A_{1}}^{T} \otimes V(g)_{C_{1}}^{\dagger}\left|\phi^{(1)}\right\rangle_{A_{1} B_{1} E_{1} C_{1}}
$$

for $n=1$. This correspondence reads that $V\left(g_{1}\right) \in \mathcal{A}$ and $V\left(g_{2}\right) \in \mathcal{B} . V(g)$ has a logical unitary operator,

$$
U(g) \otimes U(g) \otimes \cdots \otimes U(g) \otimes V(g) \in \mathcal{L}(V(g)),
$$

whose support is $B C(E C)$ if $g=\left(g_{1}, e\right)\left[g=\left(e, g_{2}\right)\right]$. Suppose the state is in a nontrivial SPT phase in the sense that $\left[V\left(g_{1}\right), V\left(g_{2}\right)\right] \neq 0$ for some $g_{1}, g_{2}$ [6]. This implies that $\mathcal{B}^{\prime} \subsetneq \mathcal{A}$. Therefore, we can reconfirm that the nontrivial $G_{1} \times G_{2}$ SPT phase implies nonzero CMI under dual complementarity.

The converse direction is entirely nontrivial. The existence of tensor-product logical unitaries $U_{B} \otimes U_{C}$ is necessary for $\phi^{(n)}$ to be a state in such a SPT phase, but this condition is not always implied by nonzero CMI, as we observe later. A particular class of $V$ in which the converse also holds is isometry comprising Clifford gates, i.e., when the MPS is a stabilizer state [15].

Theorem 2. Let $V$ be an isometry composed of Clifford gates and ancillas $|0\rangle^{\otimes k}$. Then

$$
I\left(A_{1}: C_{n} \mid B_{n}\right)_{(n)}>0 \forall n,
$$

if and only if finite groups $G_{1}$ and $G_{2}$ exist such that the MPS generated by $V$ is in a nontrivial $G_{1} \times G_{2}$ SPT phase.

The proof is given in the SM [21]. Theorem 7 can be applied for all 2D topologically trivial stabilizer states including the 2D cluster state [7]. However, the conclusion is not necessarily true outside of stabilizer states. In fact, one can find a family of boundary states such that all nonidentity logical unitaries cannot be written as $U_{B} \otimes U_{C}$.

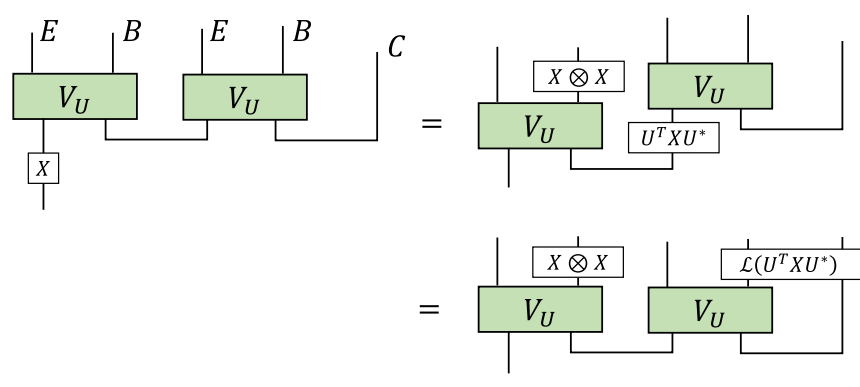

FIG. 3. A logical operator of Pauli $X$ in the example. This is a unique logical operator of $X$ supported on $B C$. For general $U$, the logical operator is no longer a tensor product of unitaries on $B$ and $C$ for $n=2$. We expect this to hold for $n>2$ as well.

\section{A NONTRIVIAL EXAMPLE}

Let $V_{U}$ be an isometry that is the Stinespring dilation of $\mathcal{E}_{U}(\sigma)=\frac{1}{4} \sum_{i=0}^{3}\left(P_{i} \otimes P_{i} U\right) \sigma\left(P_{i} \otimes U^{\dagger} P_{i}\right)$, where $P_{i} \quad(i=$ $0,1,2,3)$ are the Pauli Matrices $\left(P_{0}=I\right)$. The correctable algebras are $\mathcal{A}=\mathcal{B}=M_{2}(\mathbb{C})$; therefore, boundary states automatically satisfy dual complementarity. The CMI attains a maximum value of $I(A: C \mid B)_{(n)}=2$. Note that this model is in a $D_{2} \times D_{2}$ SPT phase if $U=I$.

For $n=1$, each Pauli operator $P_{i}$ has a unique logical operator $\left(P_{i} P_{i}\right)_{B} \otimes U^{T}\left(P_{i}\right)_{C} U^{*}{ }^{3}$ If $U$ is not a Clifford unitary nor diagonal in the $X$ or $Z$ bases, both $U^{T} X_{C} U^{*}$ and $U^{T} Z_{C} U^{*}$ are non-Pauli matrices. This induces non-tensor-product logical operators on $B_{2} C_{2}$, which are also unlikely to be a tensor product for $n>2$ (Fig. 3). By coarse-graining $\tilde{\mathcal{E}} \equiv \tilde{\mathcal{E}}^{(2)}$, we obtain a model with no logical-operator form like $U_{B} \otimes U_{C}$, but with $I(A: C \mid B)_{(n)}=2$.

We can construct a nontrivial 2D translation-invariant model by considering a layer of many copies of this $1 \mathrm{D}$ example along the vertical and the horizontal directions (decoupled stacks), as in the case of a 2D weak subsystem SPT phase [26]. The resulting 2D state has a spurious TEE for an arbitrarily large dumbbell-like region [7].

One may expect that, for a periodic boundary condition, the CMI could vanish in such a nontrivial example. Although we do not have any analytical result for this, we numerically sampled $U$ from the Haar measure and then calculated the CMI for closed chains. Figure 4 suggests that CMI remains a positive constant even for the closed boundary, while the value decreases from two.

\section{FUTURE DIRECTIONS}

A crucial open question is how to characterize or classify the nontrivial example with spurious TEE. Although it should not be in a SPT phase under the on-site $G_{1} \times G_{2}$ symmetry, it could be in a SPT phase under another type of symmetry.

Generalization to more broad classes of boundary MPS is desired. One possible extension is to consider boundary states without dual complementarity. Dual complementarity neglects all information outside of $\mathcal{A}$ but, in general, one has

\footnotetext{
${ }^{3}$ The uniqueness follows from the fact that there is no stabilizer supported on $B C$.
} 


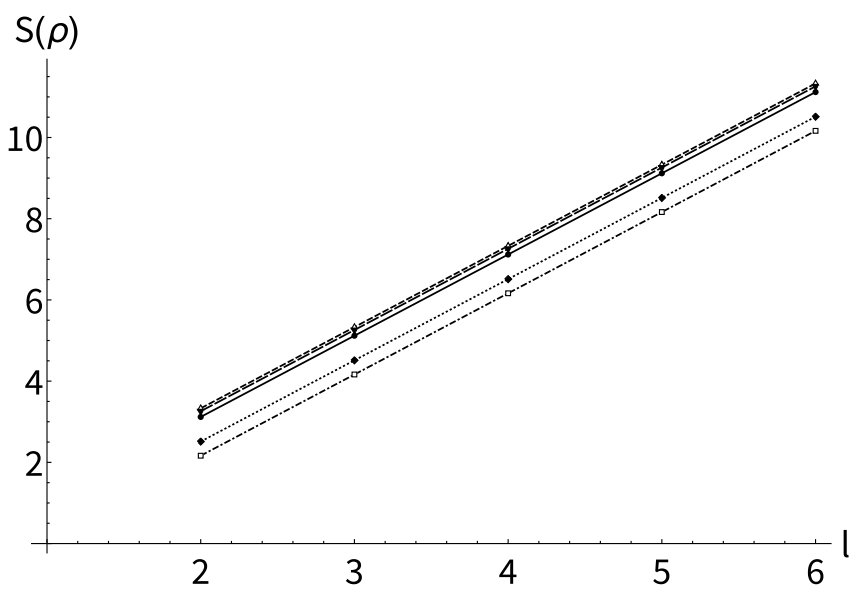

FIG. 4. The numerical result on the entropy $S\left(\rho_{A B C}\right)$ of the example with a closed boundary for five samples of $U$ from the Haar measure. $l$ is the length of the spin chain ( $l=6$ is 12-qubit). From the data, $S(\rho)=2 l-c_{0}$ with a constant $c_{o}>0$ up to $10^{-7}$ error. Since any reduced state of the example is completely mixed, it shows that $I(A: C \mid B)_{(n)}=c_{0}$ for any tripartition $A B C$ such that $B$ separates $A$ from $C$.

some "noisy" information localized on $B$ (or $E$ ). Extending the correctable algebra by adding operators carrying such information may be possible. Another important direction is considering general injective MPS including Eq. (2). We expect that general injective MPS can be decomposed into protected and unprotected parts, as in Ref. [10], such that the effect of the unprotected part vanishes exponentially as the conditioning system grows. We leave these problems for future works.

The spurious contribution exists not only in the trivial phase but also in topologically ordered phases [27]. Although the boundary MPS construction does not work in topologically ordered phases, we can construct a nontrivial example of topologically ordered models with spurious contributions by taking tensor products of any $2 \mathrm{D}$ topological order model and the models considered in this paper. Such a state could be considered as a symmetry-enriched topologically ordered phases beyond on-site symmetry.

\section{ACKNOWLEDGMENTS}

K.K. thanks Arpit Dua for a discussion about stacking 1D models to construct a 2D model. K.K. acknowledges funding provided by the Institute for Quantum Information and Matter, an NSF Physics Frontiers Center (NSF Grant PHY-1733907). F.B. is supported by the NSF. The numerical calculation in this paper was implemented in the Caltech HPC center.
[1] A. Kitaev and J. Preskill, Topological Entanglement Entropy, Phys. Rev. Lett. 96, 110404 (2006).

[2] M. A. Levin and X.-G. Wen, Detecting Topological Order in a Ground State Wave Function, Phys. Rev. Lett. 96, 110405 (2006).

[3] A. Kitaev, Fault-tolerant quantum computation by anyons, Ann. Phys. (NY) 303, 2 (2003).

[4] M. A. Levin and X.-G. Wen, String-net condensation: A physical mechanism for topological phases, Phys. Rev. B 71, 045110 (2005).

[5] B. Shi, K. Kato, and I. H. Kim, Fusion rules from entanglement, Ann. Phys. 168164 (2020).

[6] L. Zou and J. Haah, Spurious long-range entanglement and replica correlation length, Phys. Rev. B 94, 075151 (2016).

[7] D. J. Williamson, A. Dua, and M. Cheng, Spurious Topological Entanglement Entropy from Subsystem Symmetries, Phys. Rev. Lett. 122, 140506 (2019).

[8] J. Cano, T. L. Hughes, and M. Mulligan, Interactions along an entanglement cut in $2+1 \mathrm{D}$ Abelian topological phases, Phys. Rev. B 92, 075104 (2015).

[9] L. H. Santos, J. Cano, M. Mulligan, and T. L. Hughes, Symmetry-protected topological interfaces and entanglement sequences, Phys. Rev. B 98, 075131 (2018).

[10] D. T. Stephen, H. Dreyer, M. Iqbal, and N. Schuch, Detecting subsystem symmetry protected topological order via entanglement entropy, Phys. Rev. B 100, 115112 (2019).

[11] D. Perez-Garcia, F. Verstraete, M. M. Wolf, and J. I. Cirac, Matrix product state representations, Quantum Info. Comput. 7, 401 (2007).
[12] C. Beny, A. Kempf, and D. W. Kribs, Quantum error correction of observables, Phys. Rev. A 76, 042303 (2007).

[13] C. Beny, A. Kempf, and D. W. Kribs, Generalization of Quantum Error Correction via the Heisenberg Picture, Phys. Rev. Lett. 98, 100502 (2007).

[14] C. Beny, A. Kempf, and D. W. Kribs, Quantum error correction on infinite-dimensional Hilbert space, J. Math. Phys. 50, 062108 (2009).

[15] D. Gottesman, Ph.D. thesis, California Institute of Technology, 1997 (unpublished).

[16] M. B. Hastings and X.-G. Wen, Quasiadiabatic continuation of quantum states: The stability of topological ground-state degeneracy and emergent gauge invariance, Phys. Rev. B 72, 045141 (2005).

[17] X. Chen, Z.-C. Gu, and X.-G. Wen, Local unitary transformation, long-range quantum entanglement, wave function renormalization, and topological order, Phys. Rev. B 82, 155138 (2010).

[18] F. Verstraete, J. I. Cirac, J. I. Latorre, E. Rico, and M. M. Wolf, Renormalization-Group Transformations on Quantum States, Phys. Rev. Lett. 94, 140601 (2005).

[19] K. Kato, F. Furrer, and M. Murao, Information-theoretical analysis of topological entanglement entropy and multipartite correlations, Phys. Rev. A 93, 022317 (2016).

[20] J. I. Cirac, D. Pérez-Garcia, N. Schuch, and F. Verstraete, Matrix product density operators: Renormalization fixed points and boundary theories, Ann. Phys. (NY) 378, 100 (2017). 
[21] See Supplemental Material at http://link.aps.org/supplemental/ 10.1103/PhysRevResearch.2.032005 for more detailed backgrounds and the proofs of the main results.

[22] E. Knill and R. Laflamme, Theory of quantum error-correcting codes, Phys. Rev. A 55, 900 (1997).

[23] D. W. Kribs, R. Laflamme, and D. Poulin, Unified and Generalized Approach to Quantum Error Correction, Phys. Rev. Lett. 94, 180501 (2005).

[24] F. Pastawski and J. Preskill, Code Properties from Holographic Geometries, Phys. Rev. X 7, 021022 (2017).
[25] N. Schuch, D. Pérez-García, and J. I. Cirac, Classifying quantum phases using matrix product states and projected entangled pair states, Phys. Rev. B 84, 165139 (2011).

[26] Y. You, T. Devakul, F. J. Burnell, and S. L. Sondhi, Subsystem symmetry protected topological order, Phys. Rev. B 98, 035112 (2018).

[27] D. T. Stephen, J. Garre-Rubio, A. Dua, and D. J. Williamson, Subsystem symmetry enriched topological order in three dimensions, arXiv:2004.04181. 